\title{
Heme oxygenase 1 facilitates cell proliferation via the B-Raf-ERK signaling pathway in melanoma
}

\author{
Liu Liu ${ }^{1,2+}$, Yan $\mathrm{Wu}^{1,2+}$, Chunxiang Bian', Muhammad Farrukh Nisar ${ }^{1,3}$, Mei Wang ${ }^{1}$, Xiangyu Hu², \\ Qingchun Diao ${ }^{2}$, Weiqi Nian ${ }^{4}$, Enwen Wang ${ }^{4}$, Wei Xü ${ }^{2^{*}}$ and Julia Li Zhong ${ }^{1,2^{*}}$ (D)
}

\begin{abstract}
Background: Despite therapeutic advancements (e.g. B-RAF inhibitors) targeting cutaneous melanoma, many cellular processes, including inducible heme oxygenase 1 (HO-1), counteract treatments for malignancies. So there is an urgent need to find biological treatment targets, develop new therapeutic approaches and achieve longer responses. This study aimed to explore the relationship of HO- 1 and B-Raf via mediating ERK1/2 signaling on cell cycle in melanoma.

Methods: Immunohistochemistry was applied to evaluate the levels of HO-1 and B-Raf expression in melanoma tissues and adjacent healthy tissues. Co-immunoprecipitation (Co-IP) assessed the interaction of HO-1 with B-Raf. Further study overexpression and knock-down of HO-1 in A375 cell lines, especially knockout HO-1 using CRISPR-Cas9, verified HO-1 regulate cell proliferation in vivo and in vitro. Finally, Western blot analysis and qRT-PCR were performed to investigate the mechanisms by which HO-1 mediates cell cycle by B-RAF-ERK1/2 signaling.

Results: First, histology and Co-IP show that HO-1 interacts with B-Raf directly in melanoma tissue. Further study illustrated that HO-1 overexpression promotes melanoma cell proliferation while HO-1 reduction represses melanoma cell proliferation because of HO-1 affects cell cycle. Mechanistic studies revealed that HO-1 was associated with a marked activation of B-RAF-ERK1/2 signaling and led to CDK2/cyclin E activation, thereby promoting melanoma proliferation.

Conclusions: Our result reveals a previously unknown mechanism that the HO-1-B-RAF-ERK axis plays an important role in melanoma cell proliferation. Therapeutic target on $\mathrm{HO}-1$ could be a novel method for treating melanoma.
\end{abstract}

Keywords: HO-1, B-RAF, ERK1/2, Cell cycle, Melanoma

\section{Background}

Among various skin cancers, cutaneous melanoma, a malignancy originating from melanocytes, has the highest mortality. Surgery or combinational chemotherapies have been shown to confer only modest survival benefits in advanced melanoma, with a five-year survival rate below

\footnotetext{
* Correspondence: davidweixucn@gmail.com; jlzhong@cqu.edu.cn 'Liu Liu and Yan Wu contributed equally to this work. Liu Liu and Yan Wu are first authors.

2Department of Dermatology, Chongqing First People's Hospital and Chongqing Traditional Chinese Medicine Hospital, No. 40 Daomenkou St., District Yuzhong, Chongqing 400011, China

"The Base of "111 Project" for Biomechanics and Tissue Repair Engineering, Bioengineering college and Life Science College, Chongqing University, Chongqing 400044, China

Full list of author information is available at the end of the article
}

$16 \%$ in patients with metastasis [1]. Recently, the increased knowledge about the molecular mechanisms of melanoma has revolutionized its treatment. For example, the B-RAF inhibitors vemurafenib and dabrafenib have demonstrated an improvement in both overall survival and progression-free survival [2]. Unfortunately, relapse usually occurs within months after treatment [3]. Thus, there is an urgent need to develop new therapeutic approaches for melanoma treatment and prolong the responses.

Ultraviolet radiation (UVR) is estimated to be responsible for the appearance of approximately $65 \%$ of all melanomas [4], while heme oxygenase $1(\mathrm{HO}-1)$, the inducible form of heme oxygenase, can be induced in response to diverse cellular stress signals such as UVR and reactive

(c) The Author(s). 2019 Open Access This article is distributed under the terms of the Creative Commons Attribution 4.0 International License (http://creativecommons.org/licenses/by/4.0/), which permits unrestricted use, distribution, and 
oxygen species (ROS) [5]. As an enzyme with antioxidant and antiangiogenic properties, $\mathrm{HO}-1$ is usually expressed at low levels in most tissues under physiological conditions but can be highly induced following exposure to diverse chemical and physical stressors [6]. Furthermore, an increased HO-1 expression level has been reported in different cancers, including brain tumors, chronic myeloid leukemia, melanoma and lymphosarcoma, suggesting a possible contribution of $\mathrm{HO}-1$ to tumorigenesis [7-9]. These data suggest that HO-1 could is a potential biological treatment target. It is known that somatic mutations in B-RAF genes are common in melanoma. Previous studies demonstrated that $B-R A F$ is a critical gene in the development of endometriosis [10], and overexpression of wild-type B-Raf is one of the mechanisms underlying the constitutive activation of the MAPK pathway that stimulates the growth of malignant melanoma cells [11]. Moreover, a previous in vitro study showed that $\mathrm{HO}-1$ increasing in a subset of thyroid cancers is associated with tumor aggressiveness and BRAF ${ }^{\mathrm{V} 600 \mathrm{E}}$ expression [12].

Cancer is considered a disease involving cell cycle disruption. In normal cells, the cell cycle progression can be constrained, allowing the cells to discontinue cellular division under certain conditions. In contrast, the cell cycle progression is unhindered in cancer cells have unhindered. G1 phase regulation is frequently impaired in cancer cells. Thus, G1-related regulatory proteins are suitable targets for therapy. Similarly, vemurafenib, a small molecule inhibitor of a driver oncogene, binds specifically to the adenosine triphosphate (ATP) pocket of activated $\mathrm{BRAF}^{\mathrm{V} 600 \mathrm{E}}$, blocks ERK1/2 activation, halts cell cycle progression at the G0/G1 phase and promotes apoptosis. Regulation of cell cycle progression is a complex process and requires the coordinated action of cyclins, cyclin-dependent kinases (CDKs), and CDK inhibitors (CDKIs). Most tumors, including melanoma, have an abnormal G1-to-S-phase transition, mainly due to the deregulated activity of cyclin E, cyclin D, CDK2 and CDK4.

While there is a clear role of activated ERK signaling in inducing cell cycle arrest and supporting cancer cell proliferation, surprisingly little is known about the potential impact of HO-1 on the ERK signaling activity in cancers bearing oncogenic B-RAF. Here, we report a key role of HO-1 in controlling the melanoma cell cycle by regulating B-Raf expression. Endogenous HO-1 and B-Raf are highly expressed in melanoma tissues, and both are colocalized in the cytoplasm of A375 cells. Depletion of HO-1 using small interfering RNA (siRNA) or the CRISPR/Cas9-based blockade of HO-1 activity further inhibited melanoma cell proliferation, both in vitro and in vivo. The cell proliferation induced by promoting HO-1 resulted in ERK1/2 activation. Moreover, blocking HO-1 also induced cell cycle arrest at G0/G1, and overexpression of B-Raf rescued the cell cycle effect of HO-1. Our studies suggest that targeting HO-1 may be therapeutically relevant to melanoma treatments.

\section{Methods \\ Cell culture and treatment}

All cells used in the study were cultured in DMEM (HyClone) containing 10\% fetal bovine serum and maintained at $37^{\circ} \mathrm{C}$ in a humidified $5 \% \mathrm{CO}_{2}$ incubator. The medium was replaced every 2 days with fresh medium to maintain cell activity. For treatment, cells were seeded in a $60 \mathrm{~mm}$ dish. After overnight culture, cells were exposed to $\operatorname{UVR}\left(25 \mathrm{~kJ} / \mathrm{m}^{2}, 50 \mathrm{~kJ} / \mathrm{m}^{2}, 100 \mathrm{~kJ} / \mathrm{m}^{2}\right)$ or to $\mathrm{H}_{2} \mathrm{O}_{2}(40 \mathrm{mM})$ for $6 \mathrm{~h}$, and the RNA and protein were extracted $4 \mathrm{~h}$ or 10 $h$, respectively, after exposure.

\section{Tumor samples}

Tumor samples were collected from 4 consecutive patients with melanoma who underwent surgical resection at Chongqing Hospital of Traditional Chinese Medicine (Chongqing, China) between August 2016 and August 2017. Informed consent was obtained from the patients. Those patients with preoperative anticancer treatment or with evidence of other malignancies were excluded from the study. The study protocol was approved by the local Ethics Committee of Chongqing Traditional Chinese Medicine Hospital.

\section{Analysis of cell proliferation}

Cell cycle analysis was performed using fluorescence-activated cell sorting (FACS) as previously described. Additionally, clonogenic assays and the CCK- 8 assay were conducted as previously described [13].

\section{Generation of knockout cell lines using the CRISPR-Cas9 technique}

Generation of knockout cell lines using the Cas9 technique was performed as previously described [13]. The gDNA for targeting $\mathrm{HO}-1$ with the lentiCRISPRv2 vector was designed as follows: Oligo 1, 5'-CACCGGCTGCTGAC CCATGACACCA-3'; Oligo 2, 5'-AAACTGGTGTCATG GGTCAGCAGCC-3'. Protein lysates were then extracted for further analysis.

\section{Vector construction and lentiviral transduction}

The full-length coding regions of $32 \mathrm{kDa} \mathrm{HO}-1$ were amplified by PCR and cloned into the vector pLJM1 (Addgene \#19319) for expression. The specific primers were as follows: 5'-ATACCGGTCACGAACGAGCCCAGCACC-3' (forward) and 5'-GCATGCCTGAATTCACATGGCATA AAGCC-3' (reverse). To knock down endogenous HO-1, the plasmids were established with pLKO.1 (Addgene \#8453) according to the manufacturer's protocol. The target sequence was as follows: 5'-AATGCTGAGTTCAT 
GAGGAAC-3' for HO-1. Packaging of these lentiviruses was conducted as previously described [14].

\section{RNA extraction and qRT-PCR}

Total cellular RNA was extracted using TRIzol reagent (TaKaRa) following the manufacturer's protocol. RNA was reversed transcribed to cDNA using SuperScriptIII (Promega). qRT-PCR was performed with products from Applied Biological Materials Inc. (Richmond, BC, Canada) on a CFX Connect ${ }^{\text {mi }}$ Real-Time PCR Detection System (USA). Glyceraldehyde-3-phosphate dehydrogenase (GAPDH) was used as a reference gene. The relative expression levels of mRNA were quantified using the $\Delta \Delta \mathrm{Ct}$ method.

\section{Immunofluorescence and immunohistochemistry}

Immunofluorescence and immunohistochemistry staining was carried out as previously described [13]. The antibodies were B-Raf antibody (1:100, Abcam), p-ERK antibody (1:200, Cell Signaling Technology), and HO-1 antibody (1:200, Cell Signaling Technology). Slides were observed under a Nikon E600 microscope (Tokyo, Japan) with a digital camera.

\section{Western blot analysis}

Western blotting was performed as follows: the protein lysates were collected from the cells. The tissue lysates were separated on 12\% SDS-polyacrylamide gels and transferred onto polyvinylidene fluoride membranes (Bio-Rad). The antibodies used included HO-1, B-Raf, CDK2, cyclin E, phosphorylation of ERK1/2 (p-ERK) and ERK (1:1000) antibodies, while GAPDH (1:2000) was used as a loading control. The Western blot results were further analyzed using a Bio-Rad ChemiDoc ${ }^{\mathrm{mi}}$ XRS+ system.

\section{Xenografted tumor model}

All animal experiments were performed in accordance with the ethical guidelines of Laboratory Animal Welfare and Ethics Committee Of the Third Military Medical University. For the analysis of in vivo tumor growth and invasion, $3 \times 10^{7}$ A375 cells in $200 \mu$ l of DMEM were subcutaneously injected into each 4- to 6-week-old nude mouse $(n=5)$. A ruler measured the tumor size every 5 days. At 40 days or 45 days after injection, the mice were euthanized, and the tumors were surgically isolated and weighed use electronic load cell scales.

\section{Statistical analysis}

All values are expressed as the mean \pm S.D. Statistical analysis of the data was performed by a two-tailed Student's t-test $\left({ }^{*} P<0.05 ;{ }^{* *} P<0.01\right)$.

\section{Results}

HO-1 interacts with B-Raf directly in melanoma

Firstly, we found a higher HO-1 and B-Raf expression in melanoma tissue than that in adjacent healthy tissue by immunohistological analysis (Fig. 1a). In order to explore whether HO-1 and B-Raf have a regulatory relationship in melanoma cells, we assessed the expression of B-Raf in A375 cells by HO- 1 overexpression and inhibition. Results showed that overexpression of HO-1 significantly promoted the expression of B-Raf, while inhibition of HO-1 remarkably suppressed B-Raf expression (Fig. 1b and Additional file 1: Figure S1A). Additionally, we found that endogenous $\mathrm{HO}-1$ and $\mathrm{B}-\mathrm{Raf}$ are colocalized in the cytoplasm of melanoma tissue (Additional file 1: Figure $\mathrm{S} 1 \mathrm{~B})$. We then investigated whether $\mathrm{HO}-1$ could interact with B-Raf. HO-1 was transfected alone or together with B-Raf into HEK293T cells for protein-protein interaction analysis by a co-immunoprecipitation assay. As indicated in Fig. 1d \& Additional file 1: Figure S1B, HO-1 could be pulled down by B-Raf, and reversely, B-Raf could be pulled down by HO-1. Finally, we confirmed that both exogenous HO-1 and B-Raf are colocalized in the cytoplasm of A375 cells by immunofluorescence staining (Fig. 1f). Taken together, these results suggest that $\mathrm{HO}-1$ interacts with B-Raf directly.

\section{HO-1 promotes melanoma cell proliferation}

It is known that B-Raf can regulate cell growth [15], thus, we hypothesized that HO-1 could modulate cell proliferation by interacting with B-Raf in melanoma. To test this hypothesis, we first established stable A375 cell lines with HO-1 overexpression and then evaluated the cell growth using the CCK- 8 assay and a clonogenic survival assay. The overexpression efficiency was confirmed by Western blotting (Fig. 2a). We found that HO-1 overexpression resulted in an accelerated cell proliferation rate (Fig. 2a, b). In addition, we examined the cellular organization of actin filaments using phalloidin fluorescence staining. A more complex array of F-actin in HO-1-overexpressing cells was observed (Fig. 2c). Furthermore, we explored the role of HO-1 in tumorigenesis in vivo. The results show that the average size and weight of tumors significantly increased in HO-1-overexpression cells (Fig. 2d, e). These data show that HO-1 can promote melanoma cell proliferation both in vivo and in vitro.

\section{Suppression of HO-1 has an anti-proliferative effect in melanoma cells}

Then, we established stable A375 cell lines with HO-1 knockdown and found that silencing of HO-1 decreased cell proliferation rate by both CCK- 8 and a clonogenic survival assay (Fig. 3a, b and Additional file 2: Figure S2A). The results in vivo show that the average size and weight of tumors decreased in HO-1-knockdown cells (Fig. 3c and 


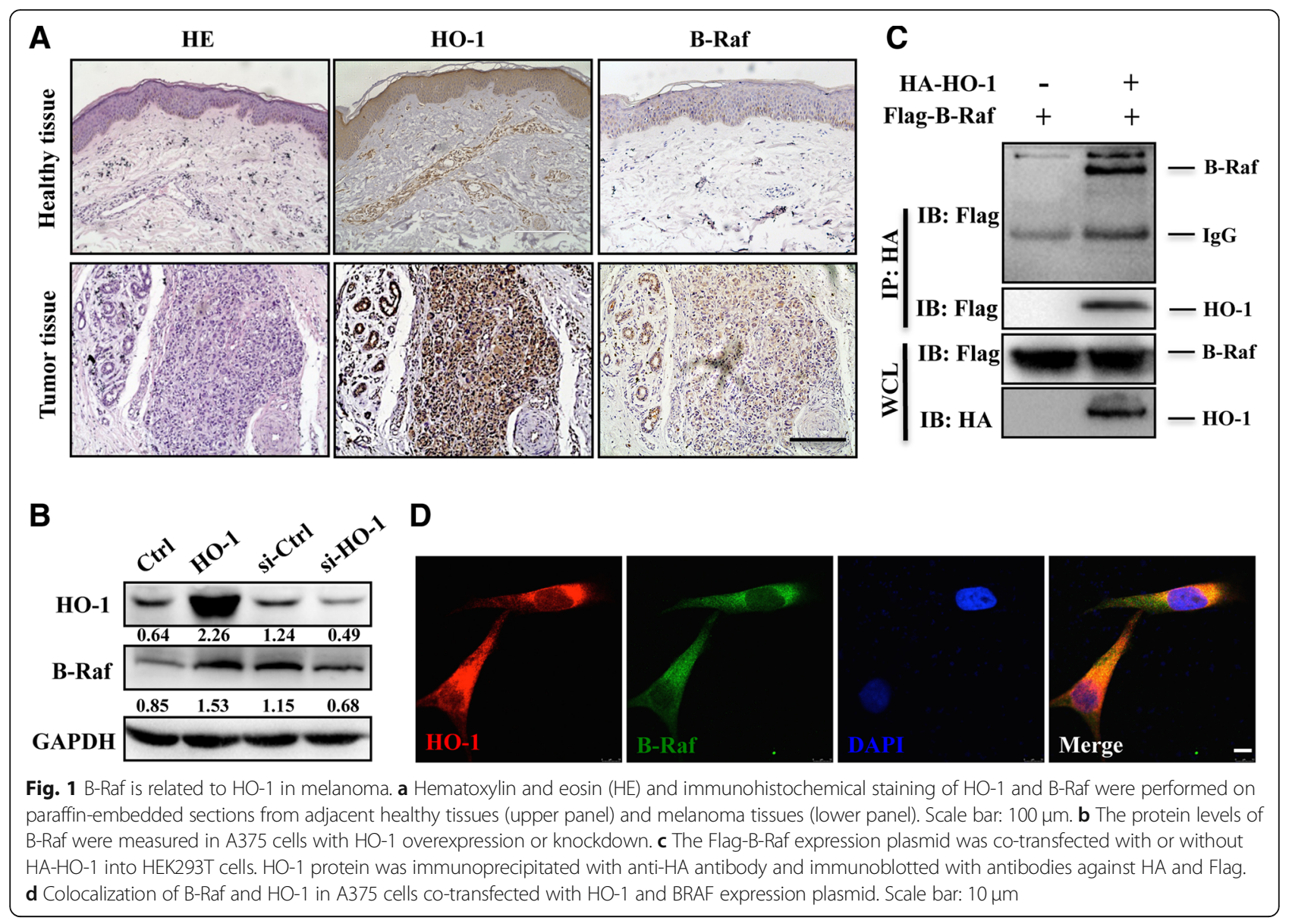

Additional file 2: Figure S2B). Since gene-silencing using the siRNA approach may not sufficient for completely knockout of HO-1, we generated a cell line (A375 HO- ${ }^{-1}$ $\left.{ }^{-}\right)$with a homozygous knockout of $\mathrm{HO}-1$ by targeting exon 4 of HO-1 using the CRISPR/Cas9 gene editing system (Fig. 3d). To verify the HO-1 knockout, we performed Western blot analyses and target site sequencing. From 8 clones, we found two homozygous mutant lines and a biallelic 1-nucleotide-inserted and 1-nucleotide-deleted line (\#5; Fig. 3e, f) and a biallelic 1-nucleotide-deleted and 9nucleotide-deleted line (\#7; Additional file 2: Figure S2C). Then, we analyzed the cellular morphology of the A375 cells and found spindle-shaped scramble control cells. In contrast, $\mathrm{HO}-1^{-/-}$cells underwent a morphological change into a cobblestone shape with more cell-to-cell contacts (Additional file 2: Figure S2D, E). In addition, we examined the cellular organization of actin filaments using phalloidin fluorescence staining. A more bundled and circumferential arrangement of $\mathrm{F}$-actin in $\mathrm{HO}^{-1} \mathrm{1}^{-/}$cells was observed, while the scramble control cells exhibited a more complex array of F-actin (Fig. 3g). The clonogenic survival assay revealed that the clone formation ability was $62.5 \%$ lower in $\mathrm{HO}_{-1}{ }^{-/-}$cells than in scramble control cells (Fig. 3h). Moreover, a CCK-8 assay revealed that the $\mathrm{HO}^{-1-}{ }^{-/}$cells grew significantly slower than the control cells (Fig. 3i).

To further evaluate the tumor suppressive ability of $\mathrm{HO}-1$ knockout in vivo, $\mathrm{HO}-1^{-/-}$cells were injected subcutaneously into severe combined immunodeficiency (SCID) mice. The tumor volumes resulting from the $\mathrm{HO}_{-} \mathrm{-}^{-1-}$ cells were significantly smaller than the tumor volumes resulting from the scramble control cells (Fig. 3j). The tumor growth curves for a five-day period show that the xenograft tumors of $\mathrm{HO}-1^{-1-}$ cells grew significantly slower than the scramble control cells (Fig. 3j). At the endpoint of the tumor growth experiments at day 45 , the tumors from each group were excised and weighed. The HO-1 knockout tumors were much lighter than the control tumors (Fig. 3k). These results suggest that the lack of HO-1 reduces the tumor growth of A375 cells in vivo and vitro.

\section{HO-1 facilitates the progression of the cell cycle through B-Raf}

To further understand the molecular mechanisms linking HO-1 and melanoma cell viability, we assessed cell 


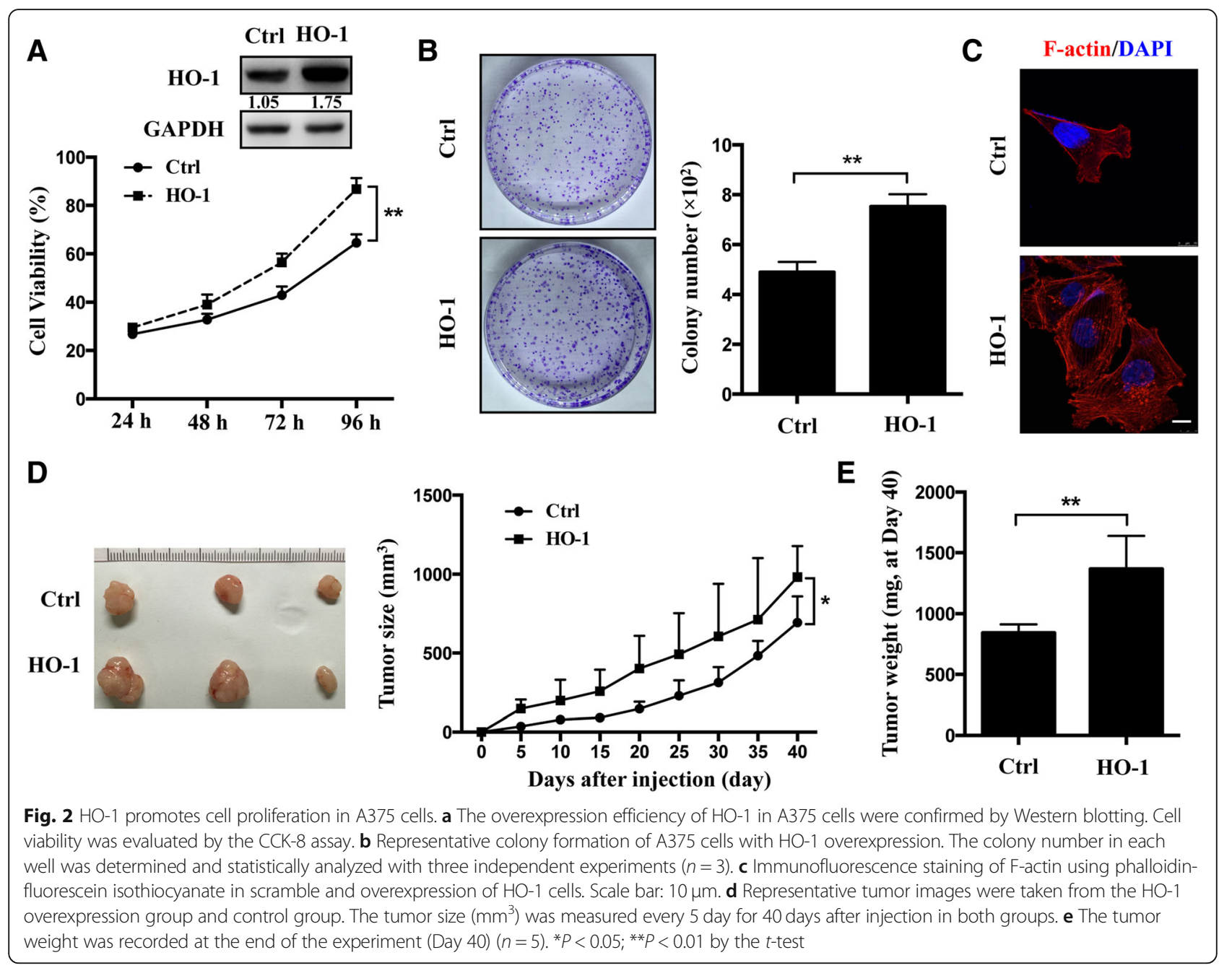

cycle distributions by flow cytometry. As shown in Fig. 4a, HO-1-overexpression in A375 cells demonstrated a significant decrease in the percentage of cells in the G1 phase and an increase in the percentage of cells in the $\mathrm{S}$ phase. Conversely, a decrease in the cell percentage in the $S$ phase and an increase in the percentage in the $\mathrm{G} 1$ phase were observed in the $\mathrm{HO}-1$ knockout and HO-1-silenced A375 cells (Fig. 4b and Additional file 3: Figure S3A). A number of key markers, such as CDKs (CDK2, CDK4, CDK2) and cyclin (B, D, $\mathrm{E})$, have been reported to modulate the cell cycle dynamically. We noticed that among these molecules, cyclin $\mathrm{E}$ and $\mathrm{CDK} 2$ had markedly increased levels after HO-1 overexpression (Fig. 4c and Additional file 3: Figure S3B). As expectedly, the level was decreased after eliminating or silencing of $\mathrm{HO}-1$ (Fig. $4 \mathrm{~d}$ and Additional file 3: Figure S3C, D). Consistent with these results, the RT-PCR results also showed significant downregulation of cyclin $\mathrm{E}$ and $\mathrm{CDK} 2$ in the $\mathrm{HO}-1^{-/-}$xenograft tumors (Fig. 4e). It is well known that HO-1 can be induced upon exposure to oxidative stress, such as UVR and $\mathrm{H}_{2} \mathrm{O}_{2}$. We then asked whether the induced HO-1 in response to external stimuli is associated with the cell cycle. We found that, along with HO-1, UV induces B-Raf expression in melanoma cells (A375) as well as the cell cycle markers (Fig. 4f). Similarly, the protein level of B-Raf was also increased after treatment with $\mathrm{H}_{2} \mathrm{O}_{2}$ (Fig. 4g). These results suggest that HO-1 facilitates the progression of the cell cycle by promoting the expression of cyclin E and CDK2.

Since we already knew that $\mathrm{HO}-1$ could interact with B-Raf directly, here, we asked whether the HO-1- promote cell cycle through cyclin E and CDK2, is associated with B-Raf. For this purpose, A375 cells were transfected with B-Raf vectors. Cell cycle analysis showed that $\mathrm{HO}-1$ reduction led to substantial accumulation in the G0/G1 phase by A375 cells transfected with B-Raf (Fig. 5a), and this change was accompanied by increases in the mRNA and protein levels of cyclin $\mathrm{E}$ and $\mathrm{CDK} 2$ (Fig. 5b, c). In addition, the $\mathrm{HO}-1^{-1-}$ cells were transfected with B-Raf vectors. B-Raf overexpression not only rescued the $\mathrm{HO}-1^{-/-}$cell morphological changes (Fig. 5d) but 
also rescued the cell cycle distribution and the related regulator genes (cyclin $\mathrm{E}$ and CDK2) (Fig. 5e, f). In summary, HO-1 mediated B-Raf expression and then increased the expression of cyclin E/CDK2, which are involved in regulating the cell cycle and cell proliferation in melanoma.
Involvement of p-ERK signaling in HO-1-induced proliferation

A previous study found that the RAF-ERK pathway is one of the key signal transduction pathways that participate in the cell cycle [16]. The findings raised the question of HO-1 contributes to the cell cycle by regulating

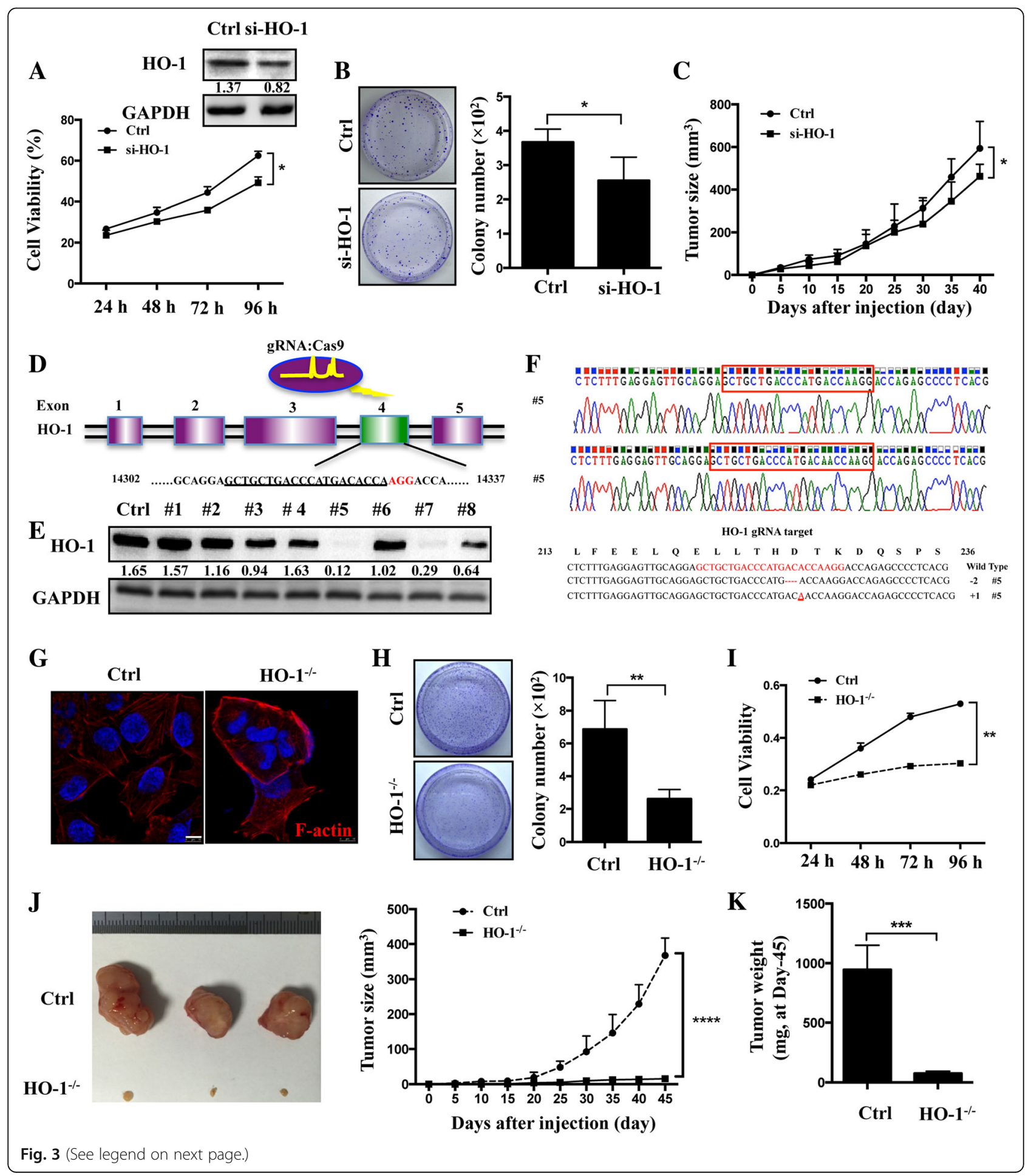


(See figure on previous page.)

Fig. 3 Inhibition of HO-1 represses A375 cell proliferation. a The knockdown efficiency of HO-1 in A375 cells was confirmed by Western blotting at 36 $\mathrm{h}$ after transfection. Cell viability was evaluated by the CCK-8 assay. b Representative colony formation of A375 cells with HO-1 knockdown. The colony number in each well was determined and statistically analyzed with three independent experiments $(n=3)$. c The tumor size $\left(\mathrm{mm}^{3}\right)$ was measured every 5 day for 40 days after injection in HO-1 knockdown group and control group. $\mathbf{d}$ Schematic representation of the genomic DNA structure of the HO-1 gene. The sequences, which include the AGG PAM targeted by gRNA with the CRISPR-Cas9 system, is shown in red. e The HO-1 expression in the knockout cell clone candidates was evaluated by Western blotting. $\mathbf{f}$ Clone No. 5 had the highest degree of HO-1 reduction and was sequenced, and the sequence was aligned with the wild-type sequence (+: inserted bases; -: deleted bases). $\mathbf{g}$ Immunofluorescence staining of F-actin using phalloidin-fluorescein isothiocyanate in scramble and $\mathrm{HO}_{-1}{ }^{-/}$cells. Scale bar: $10 \mu \mathrm{m}$. $\mathbf{h}$ The colony number in each well was determined and statistically analyzed to evaluate the clonogenic survival. i CCK-8 assays were used to determine the cell viability of the HO-1 $1^{-1-}$ cells after treatment. j A375 cells with or without CRISPR/Cas9 HO-1 knockout were subcutaneously injected into the flanks of 6-week-old female SCID mice. Representative tumor images were taken. Tumor volumes $\left(\mathrm{mm}^{3}\right)$ were measured every 5 days for 45 days. $\mathbf{k}$ Tumors were weighed at the endpoint of experiment (Day 45). ${ }^{* *} P<0.01 ;{ }^{* * *} P<0.001$ by the $t$-test

p-ERK signaling. To evaluate this possibility, we first analyzed human biopsies using immunohistological staining. The results show dramatically higher p-ERK expression in melanoma tissues than that in the adjacent healthy tissue (Fig. 6a). Furthermore, overexpression of HO-1 resulted in increased p-ERK in A375 cells. In addition, silencing or knockout of HO-1 induced downregulation of p-ERK (Fig. 6b, c). Consistent with these results, immunofluorescence staining also shows upregulation of p-ERK in HO-1-overexpressing cells, while downregulation of $\mathrm{p}$-ERK in $\mathrm{HO}-1^{-1-}$ cells (Fig. 6d). In addition, $\mathrm{HO}-1^{-1-}$ cells transfected with $\mathrm{B}-\mathrm{Raf}$ led to greater promotion of p-ERK (Fig. 6e). Interestingly, in A375 cells transfected with B-Raf, the p-ERK and B-Raf levels clearly decreased after treatment with the HO-1 inhibitor ZnPPIX, suggesting that HO-1 is the predominant mediator of MAPK reactivation in the presence of B-Raf (Fig. 6f). To probe the involvement of p-ERK activation by HO-1 in the A375 cell cycle, B-Raf overexpression was performed. Overexpression of B-Raf not only activated ERK signaling but also regulated cyclin E and CDK2 (Fig. 6g). Notably, cyclin $\mathrm{E}$ and CDK2, which are required for p-ERK deactivation by the MAPK inhibitor (PD98059), were also suppressed, while $\mathrm{HO}-1$ overexpression rescued the decrease in cell cycle proteins by p-ERK deactivation (Fig. 6h). These results reveal that the HO-1- promote cell proliferation may be mainly mediated via cyclin E/CDK2, through activation of the B-Raf/ERK pathway in melanoma.

\section{Discussion}

Melanomas arise from the pigment-producing cells called melanocytes, which normally reside in the epiderma and extend their dendrites into keratinocytes for melanin transportation and other cell-cell contacts [4]. Melanocytes also interact with skin fibroblasts and basal cells [17]. Thus, many researchers believe that the microenvironment of melanocytes plays an important role in melanoma development [18]. While melanocytes are located in a relatively deep layer of the epidermis, whether UVR causes melanoma has been debated for the past decades [19]. HO-1 is a potent anti-inflammatory intracellular mediator that catalyzes the degradation of heme to iron, biliverdin, and carbon monoxide (CO) [20]. High level of HO-1 expression is observed in many types of cells by stimuli i.e. UVR radiation [21]. Indeed, we found significantly increased HO-1 expression in A375 cells treated with UV. Thus far, numerous data indicate that HO-1 level is high in multiple cancer cells, including sarcoma, melanoma, and pancreatic cancer cell lines [22]. Additionally, the upregulation of HO-1 in melanoma cancer cells is accompanied by the increased production of VEGF associated with cancer development and progression [23]. Similarly, our study showed higher HO-1 expression in skin melanoma tissues. However, studies on the functional role of HO-1 in melanoma are rarely reported, and its function with respect to tumorigenesis and development is poorly characterized. In the current study, we clarified the cause and relationship between HO-1 and B-RAF in terms of the cell proliferation in A375 melanoma cells.

$B-R A F$ is a proto-oncogene that is also known as v-raf murine sarcoma viral homolog $B 1$, which belongs to the Raf kinase family [24]. B-RAF mutations are associated with numerous types of malignant tumors, where the mutants activate the MAPK signaling pathway constitutively, resulting in uncontrolled cell proliferation and survival [25]. However, other malignant tumors, including primary uveal melanoma and uveal melanoma, demonstrate a lack of $B-R A F$ mutations [26]. Some researchers found no mutations in the hotspot regions of $B-R A F$ (i.e., exon 15) in primary clear cell ovarian carcinoma [27]. It has been suggested that B-RAF plays an important role in tumor development by binding to specific molecular signaling molecules. Previous studies demonstrated that $B-R A F$ is a candidate gene for the development of endometriosis [10], and overexpression of wild-type B-Raf is one of the causes underlying the constitutive activation of the MAPK pathway that stimulates the growth of malignant melanoma cells [11]. Another study performed by our group found that the expression of ${ }^{\mathrm{wt}} \mathrm{B}$-Raf was also promoted in melanoma cells (A375, with $\mathrm{BRAF}^{\mathrm{V} 600 \mathrm{E}}$ mutations) after UV 


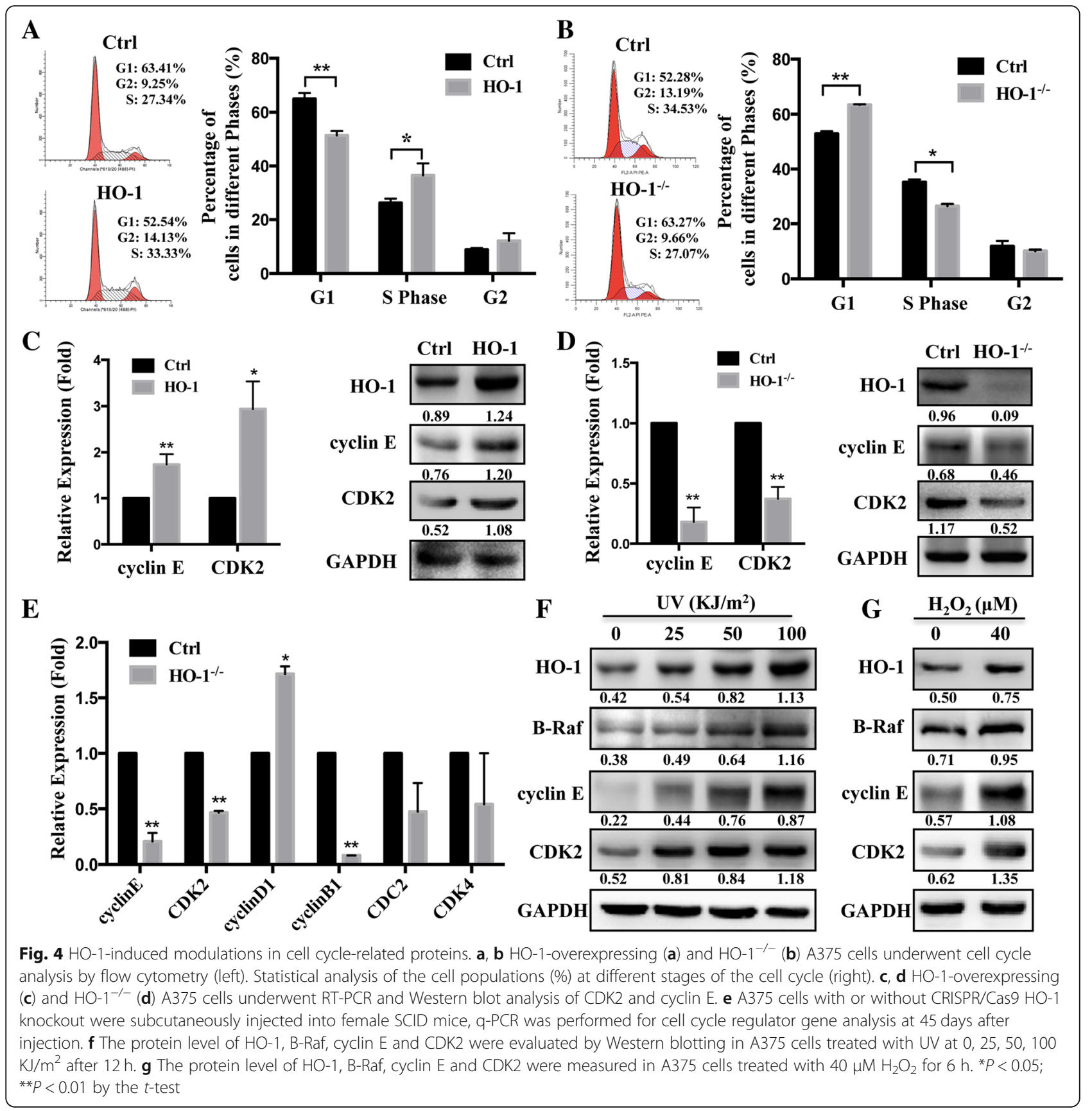

treatment, and the results showed that similar to HO-1, ${ }^{\mathrm{wt}} \mathrm{B}-\mathrm{Raf}$ maintain a high level in skin melanoma tissues. These results led us to propose that HO-1 is related to ${ }^{\mathrm{wt}} \mathrm{B}$-Raf in mutant melanoma cells. Our results finally revealed that HO-1 overexpression or knockdown is correlated with the ${ }^{\mathrm{wt}} \mathrm{B}-\mathrm{RAF}$ level in melanoma, and the connection is even more apparent in $\mathrm{HO}-1^{-/-}$cells. Furthermore, the present study found that $\mathrm{HO}-1$ physically interacts with B-RAF and promotes its kinase activity in A375 cells. These results suggest that B-RAF plays a role downstream of HO-1 in promoting cancer development.
It was known that B-RAF contributes to cell growth by regulating the cell cycle [28], and one implication of the observations reported here is that $\mathrm{HO}-1$ may be involved in regulating cell proliferation. Further research by overexpression and knockdown of HO-1 showed that HO-1 promotes cell growth in vivo and in vitro. However, it has been suggested that gene silencing by using a siRNA approach is not sufficient for an in-depth study of the molecular mechanisms of a gene [29, 30]. Furthermore, we used a CRISPR/Cas9 approach to knock out the HO-1 gene in A375 cells, this approach enabled a 


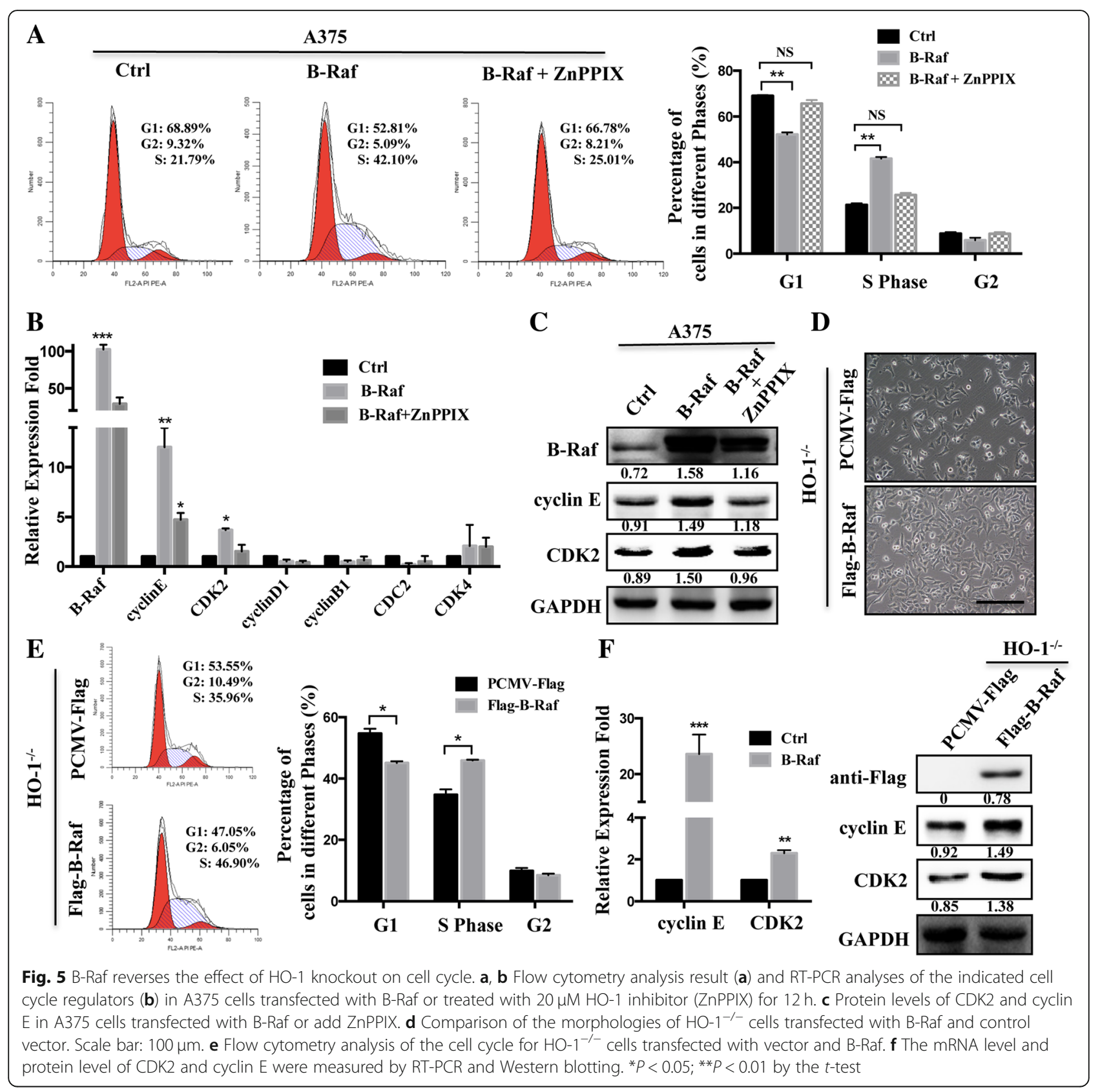

comprehensive analysis of the role of $\mathrm{HO}-1$ and provided strong evidence that $\mathrm{HO}-1$ exerts promoter effects in A375 cells. Interestingly, the $\mathrm{HO}-1^{-1-}$ cells showed a typical morphological phenotype (spindle shaped to cobblestone cell shape change) that was not observed in previous experiments with si-HO-1 where the functional depletion of HO-1 might be insufficient. In addition, the number of A375 cells in S phase of the cell cycle was significantly lower after HO-1 was blocked, while HO-1 overexpression increased this number. Our study revealed molecular mechanisms in which $\mathrm{HO}-1$ induces cell arrest at G0/G1 and showed that genetic depletion of HO-1 exerts antitumor effects. CDKs are protein kinases that regulate the cell cycle by binding to cyclin [31, 32]. The CDK2-cyclin E complex is a major player in cell proliferation that is required for progression through G1 phase and entry into $S$ phase of the cell cycle [33]. Moreover, CDK2 depletion suppresses cell cycle progression in melanoma cells [34]. Here, we noted that knockdown and knockout of HO-1 in melanoma cells significantly downregulated both CDK2 and cyclin E protein levels, whereas HO-1 overexpression could reverse these effects on CDK2 and cyclin E. Finally, via rescue methods in $\mathrm{HO}^{-1 /-}$ cells, this study provided proof that the $\mathrm{HO}^{-1-}$ cells induced changes in the cell cycle through the cyclin E and CDK2 regulatory proteins were associated with B-Raf expression. 


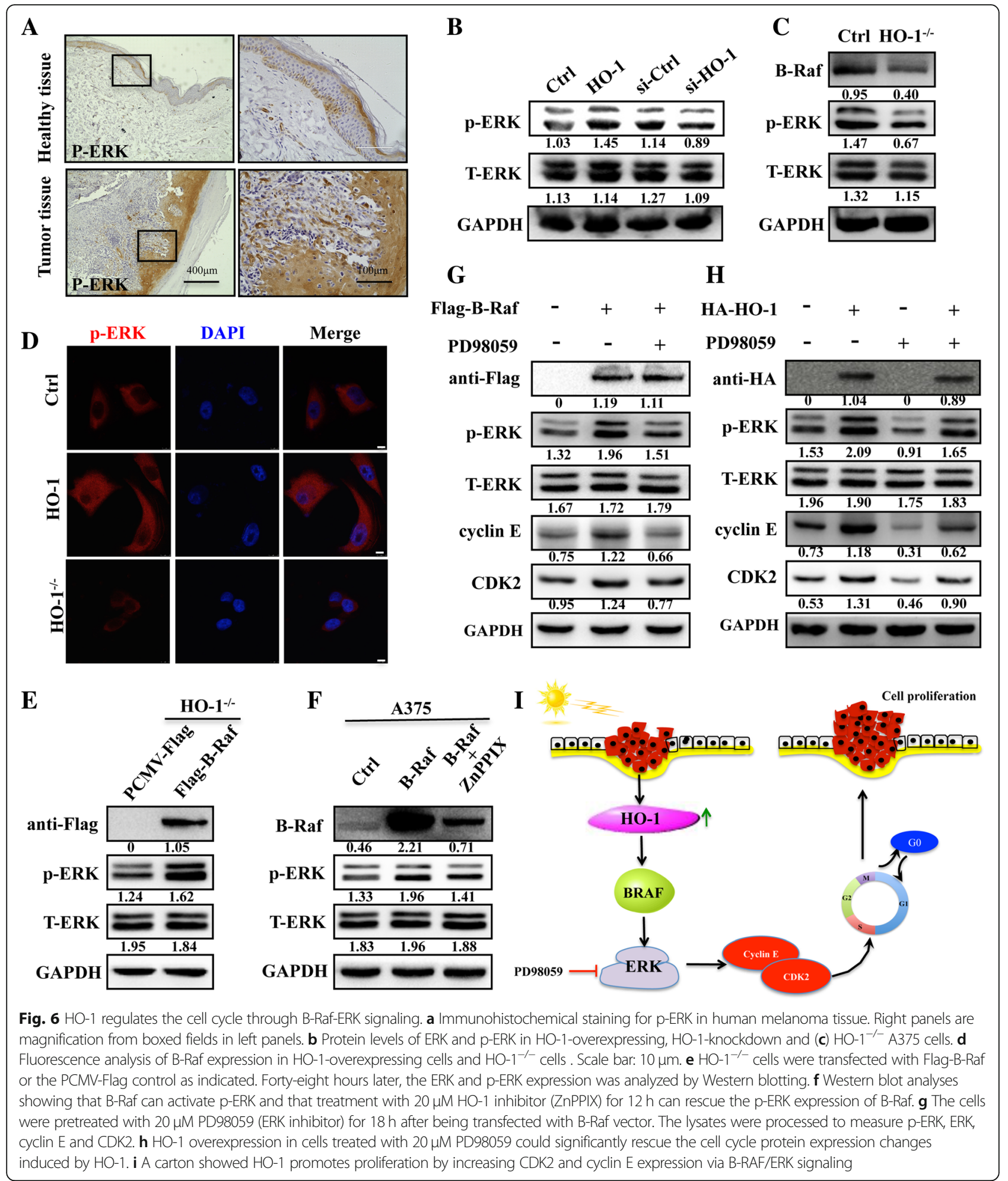

The above results indicate that the HO-1-regulated ${ }^{w t} \mathrm{~B}-\mathrm{RAF}$ expression may lead to an increase in the levels of the CDK2-cyclin E complex that stimulates the G1/S transition, which is probably involved in HO-1-induced melanoma cell proliferation.
Some investigators have studied the downstream signaling pathways of B-RAF and found that the RAS/RAF/MAPK pathway is a critical step in the initiation of melanocyte neoplasias [35]. Furthermore, the identification of factors that modulate the balance in favor of ERK may provide additional 
targets for effective therapy [36]. Our data also confirmed the ERK1/2 was activated in melanoma tissue, as found in previous research [37]. Given that the effects of B-RAF inhibition on cell viability are ERK dependent, we postulate that HO-1-promoted cell growth is mediated through ERK activation. In line with previous studies, a major study suggested the ERK1/2 kinase pathways mediated HO-1 activation and strongly supported the role of HO-1 in directly quenching oxidative stress, normalizing intracellular redox balance, and enhancing cellular survival [38]. Interestingly, HO-1, as an upstream molecule, may promote cell cycle changes via a B-RAF-ERK1/2-dependent mechanism. In addition, suppressing ERK by PD98059 may result in a marked downregulation of CDK2/cyclin E. In contrast, $\mathrm{CDK} 2 /$ cyclin $\mathrm{E}$ was restored in the cells transfected with HO-1. This finding may be associated with the activation of B-RAF/ERK signaling, which rescues the G1 cell cycle arrest and promotes cell proliferation by modulating the G1/S transition while enhancing CDK2/cyclin E activities.

\section{Conclusions}

In conclusion, as summarized in Fig. 6i, the present study demonstrates that $\mathrm{HO}-1$ promotes cell proliferation via the B-Raf-ERK signaling pathway in melanoma. HO-1 could be a potential novel anticancer target for melanoma therapy.

\section{Additional files}

Additional file 1: Figure S1. B-Raf interacts with $\mathrm{HO}-1$ directly. (A) The mRNA levels of the B-Raf were detected in A375 cells with HO-1 over-expression or knockdown. (B) Colocalization of B-Raf and HO-1 in adjacent healthy tissues and melanoma tissues. The cellular localization of HO-1 (red) and B-Raf (green) was examined by immunofluorescence staining with the corresponding antibodies. Nuclear DNA was stained with DAPI (blue). Scale bar: $100 \mu \mathrm{m}$. (C) HA-HO-1 expression plasmid was cotransfected with or without Flag-B-Raf into HEK293T cells. B-Raf protein was immunoprecipitated with anti-Flag antibody, and immunoblotted with antibodies against HA and Flag. The expression of B-Raf and HO-1 in whole-cell lysate (WCL) were confirmed. (TIF $914 \mathrm{~kb}$ )

Additional file 2: Figure S2. Knockout of HO-1 repressed A375 cell proliferation. (A) Morphological changes in cells with HO-1 knockdown compared to control cells. Scale bar: $100 \mu \mathrm{m}$. (B) Representative tumor images were taken from the HO-1 knockdown group and control group. The tumor weight was recorded at the end of the experiment (Day 40) $(n=5) .{ }^{*} P<0.05$; ${ }^{*} P<0.01$ by the $t$-test. (C) Sequencing results for Clone \#7. Clone \#7 showed the highest degree of HO-1 reduction and was sequenced, and the sequence was aligned with the wild-type sequence (-: deleted bases). (D) Morphological changes in cells with HO-1 knockout compared to control cells. Compared with scramble control cells, cells with HO-1 knockout had more cell-cell contacts. Scale bar: 100 um. (E) Morphological changes of one cell grown for 4 days for a comparison between the scramble cells and HO-1-/- cells. Scale bar: 50 um. (TIF $7670 \mathrm{~kb}$ )

Additional file 3: Figure S3. $\mathrm{HO}-1$ regulated cell cycle-related proteins. (A) A375 cells with HO-1 knockdown cell cycle analysis by flow cytometry (left). Statistical analysis of the cell populations (\%) at different stages of the cell cycle (right). All shown values are the mean \pm SD of three measurements, which were repeated three times with similar results. (B) HO-1-overexpressing A375 cells underwent RT-PCR analysis for cyclin D1, cyclin B1, CDK4 and CDC2. (C) mRNA levels and protein levels of several cell cycle makers in CDC2 were determined by RT-PCR in scramble control and HO-1-/- cells. ${ }^{*} P<0.05$; ${ }^{*} P<0.01 ;{ }^{*} P<0.001$ by the t-test. (TIF $\left.914 \mathrm{~kb}\right)$

\section{Abbreviations}

ATP: Adenosine triphosphate; CCK-8: Cell counting kit; CDKIs: CDK inhibitors; CDKs: Cyclin-dependent kinases; CO: Carbon monoxide; Co-IP: Coimmunoprecipitation; crispr/cas9: Clustered Regularly Interspaced Short Palindromic Repeats (Cas9 nuclease-null); FACS: Fluorescence-activated cell sorting; $\mathrm{H}_{2} \mathrm{O}_{2}$ : Hydrogen peroxide; $\mathrm{HO}-1$ : Heme oxygenase $1 ; \mathrm{HO}^{-1-}$ : HO-1 knockout cell; MAPK: Mitogen-activated protein kinase; ROS: Reactive oxygen species; SCID: Severe combined immunodeficiency; UVR: Ultraviolet radiation; ZnPPIX: Zn-protoporphyrin-IX

\section{Acknowledgements}

The authors thank Prof. Jiankang Liu (Xi'an Jiaotong University) and Jörg W. Bartsch for their helpful comments and suggestions on the manuscript.

\section{Funding}

This work was supported by the National Natural Science Foundation of China under Grant 81570703, the National Science Foundation for Young Scholars $(81704090,81503588)$,and the Natural Science Foundation of Chongqing (cstc2015jcyjA10113, cstc2017jcyjbx0044). Both projects are not in a financial or personal relationship to this study.

\section{Availability of data and materials}

All data generated in this study are included in the manuscript.

\section{Authors' contributions}

$L L, Y W$ and CXB performed the experiments. XYH helped with mouse breeding and flow cytometry. WQN and EWW provided specific reagents and human skin samples. LL, YW, WX, QCD designed the conceptual idea for this study. YW, WX, JLZ and MFN wrote the manuscript. All the authors approved the submission of this manuscript in its final form.

\section{Ethics approval and consent to participate}

All animal procedures were performed under the ethical guidelines of Laboratory Animal Welfare and Ethics Committee Of the Third Military Medical University and according to the recommendations of the Chongqing Experimental Animal Regulation Board (SCXK-PLA-20140011); the approval number is SYXK-PLA-20140031.

\section{Consent for publication}

All presentations of case reports have consent for publication.

\section{Competing interests}

The authors declare that they have no competing interests.

\section{Publisher's Note}

Springer Nature remains neutral with regard to jurisdictional claims in published maps and institutional affiliations.

\section{Author details}

"The Base of "111 Project" for Biomechanics and Tissue Repair Engineering, Bioengineering college and Life Science College, Chongqing University, Chongqing 400044, China. ${ }^{2}$ Department of Dermatology, Chongqing First People's Hospital and Chongqing Traditional Chinese Medicine Hospital, No. 40 Daomenkou St., District Yuzhong, Chongqing 400011, China. ${ }^{3}$ Department of Physiology and Biochemistry, Cholistan University of Veterinary and Animal Sciences (CUVAS), Bahawalpur 63100, Pakistan. ${ }^{4}$ Chongqing Cancer Institute, Chongqing University Cancer Hospital, Chongqing 400030, China.

Received: 31 August 2018 Accepted: 20 December 2018

Published online: 11 January 2019

\section{References}

1. Rozeman EA, Dekker TJA, Haanen J, Blank CU. Advanced melanoma: current treatment options, biomarkers, and future perspectives. Am J Clin Dermatol. 2018;19(3):303-17.

2. Flaherty KT, Puzanov I, Kim KB, et al. Inhibition of mutated, activated BRAF in metastatic melanoma. New England J Med. 2010;363(9):809-19.

3. Sosman JA, Kim KB, Schuchter $L$, et al. Survival in BRAF V600-mutant advanced melanoma treated with vemurafenib. New England J Med. 2012; 366(8):707-14 
4. Emri G, Paragh G, Tosaki A, et al. Ultraviolet radiation-mediated development of cutaneous melanoma: An update. J Photochem Photobiol B. 2018;185:169-75.

5. Li Volti G, Galvano F, Scacco A, et al. Anesthetics and natural heme oxygenase-1 inducers: waiting for carbon monoxide? Ann Surg. 2008;247(4): 712-3 author reply 713-714.

6. Son Y, Lee $\mathrm{JH}$, Chung HT, Pae HO. Therapeutic roles of heme oxygenase-1 in metabolic diseases: curcumin and resveratrol analogues as possible inducers of heme oxygenase-1. Oxidative Med Cell Longev. 2013:2013:639541

7. Vashist YK, Uzunoglu G, Deutsch L, et al. Heme oxygenase-1 promoter polymorphism is a predictor of disease relapse in pancreatic neuroendocrine tumors. J Surg Res. Apr 2011;166(2):e121-7.

8. Zhu X, Fan WG, Li DP, Lin MC, Kung H. Heme oxygenase-1 system and gastrointestinal tumors. World J Gastroenterol. 2010;16(21):2633-7.

9. Jozkowicz A, Was H, Dulak J. Heme oxygenase-1 in tumors: is it a false friend? Antioxid Redox Signal. 2007;9(12):2099-117.

10. Chen Q, Zhang C, Chen Y, Lou J, Wang D. Identification of endometriosisrelated genes by representational difference analysis of cDNA. Aust N Z J Obstet Gynaecol. 2012;52(2):140-5.

11. Tanami H, Imoto I, Hirasawa A, et al. Involvement of overexpressed wildtype BRAF in the growth of malignant melanoma cell lines. Oncogene. 2004;23(54):8796-804.

12. Wang TY, Liu CL, Chen MJ, Lee JJ, Pun PC, Cheng SP. Expression of haem oxygenase-1 correlates with tumour aggressiveness and BRAF V600E expression in thyroid cancer. Histopathology. 2015;66(3):447-56.

13. Wu Y, Bian C, Zhen $C$, et al. Telomerase reverse transcriptase mediates EMT through NF-kappaB signaling in tongue squamous cell carcinoma. Oncotarget. 2017;8(49):85492-503.

14. Bian C, Zhong M, Nisar MF, et al. A novel heme oxygenase-1 splice variant, $14 \mathrm{kDa} \mathrm{HO}-1$, promotes cell proliferation and increases relative telomere length. Biochem Biophys Res Commun. 2018;500(2):429-34.

15. Han S, Ren Y, He W, et al. ERK-mediated phosphorylation regulates SOX10 sumoylation and targets expression in mutant BRAF melanoma. Nat Commun. 2018;9(1):28

16. Niessner $\mathrm{H}$, Sinnberg $\mathrm{T}$, Kosnopfel $\mathrm{C}$, et al. BRAF Inhibitors Amplify the Proapoptotic Activity of MEK Inhibitors by Inducing ER Stress in NRASMutant Melanoma. Clin Cancer Res. 2017;23(20):6203-14.

17. Jobe NP, Zivicova V, Mifkova A, et al. Fibroblasts potentiate melanoma cells in vitro invasiveness induced by UV-irradiated keratinocytes. Histochem Cell Biol. May 2018;149(5):503-16.

18. Sample A, He YY. Mechanisms and prevention of UV-induced melanoma. Photodermatology, photoimmunology \& photomedicine. 2018;34(1):13-24.

19. Liu L, Zhang W, Gao T, Li C. Is UV an etiological factor of acral melanoma? J Expo Sci Environ Epidemiol. 2016;26(6):539-45

20. Maines MD. New insights into biliverdin reductase functions: linking heme metabolism to cell signaling. Physiology. 2005;20:382-9.

21. Xiang Y, Liu G, Yang L, Zhong JL. UVA-induced protection of skin through the induction of heme oxygenase-1. Bioscience trends. 2011;5(6):239-44.

22. Waza AA, Hamid Z, Ali S, Bhat SA, Bhat MA. A review on heme oxygenase-1 induction: is it a necessary evil. Inflammation Res. 2018:67(7):579-88.

23. Was $H$, Cichon T, Smolarczyk R, et al. Overexpression of heme oxygenase-1 in murine melanoma: increased proliferation and viability of tumor cells, decreased survival of mice. Am J Pathol. 2006;169(6):2181-98.

24. Ikawa S, Fukui M, Ueyama Y, Tamaoki N, Yamamoto T, Toyoshima K. B-raf, a new member of the raf family, is activated by DNA rearrangement. Mol Cell Biol. 1988;8(6):2651-4.

25. Sclafani F, Gullo G, Sheahan K, Crown J. BRAF mutations in melanoma and colorectal cancer: a single oncogenic mutation with different tumour phenotypes and clinical implications. Crit Rev Oncol Hematol. 2013;87(1):55-68.

26. Rimoldi D, Salvi $\mathrm{S}$, Lienard $\mathrm{D}$, et al. Lack of BRAF mutations in uveal melanoma. Cancer Res. 2003;63(18):5712-5.

27. Zannoni GF, Improta G, Chiarello G, et al. Mutational status of KRAS, NRAS, and BRAF in primary clear cell ovarian carcinoma. Virchows Arch. 2014; 465(2):193-8.

28. Xu W, McArthur G. Cell cycle regulation and melanoma. Curr Oncol Rep. 2016;18(6):34.

29. Wu C, Li J, Wang W, Hammond PT. Rationally Designed Polycationic Carriers for Potent Polymeric siRNA-Mediated Gene Silencing. ACS nano. 2018;12(7):6504-14.
30. Mollanoori H, Teimourian S. Therapeutic applications of CRISPR/Cas9 system in gene therapy. Biotechnol Lett. 2018:40(6):907-14.

31. Sherr CJ, Roberts JM. Living with or without cyclins and cyclin-dependent kinases. Genes Dev. 2004;18(22):2699-711.

32. Miele L. The biology of cyclins and cyclin-dependent protein kinases: an introduction. Methods Mol Biol. 2004;285:3-21.

33. Otto T, Sicinski P. Cell cycle proteins as promising targets in cancer therapy. Nat Rev Cancer. 2017;17(2):93-115.

34. Du J, Widlund HR, Horstmann MA, et al. Critical role of CDK2 for melanoma growth linked to its melanocyte-specific transcriptional regulation by MITF. Cancer Cell. 2004:6(6):565-76.

35. Lei FX, Jin L, Liu XY, et al. RIP1 protects melanoma cells from apoptosis induced by BRAF/MEK inhibitors. Cell Death Dis. 2018;9(6):679.

36. Mirmohammadsadegh A, Mota R, Gustrau A, et al. ERK1/2 is highly phosphorylated in melanoma metastases and protects melanoma cells from cisplatin-mediated apoptosis. J Invest Dermatol. 2007;127(9):2207-15.

37. Sun $X$, Shi B, Zheng $H$, et al. Senescence-associated secretory factors induced by cisplatin in melanoma cells promote non-senescent melanoma cell growth through activation of the ERK1/2-RSK1 pathway. Cell Death Dis. 2018:9(3):260.

38. Feng XE, Liang TG, Gao J, Kong P, Ge R, Li QS. Heme Oxygenase-1, a Key Enzyme for the Cytoprotective Actions of Halophenols by Upregulating Nrf2 Expression via Activating Erk1/2 and PI3K/Akt in EA.hy926 Cells. Oxid Med Cell Longev. 2017;2017:7028478.
Ready to submit your research? Choose BMC and benefit from:

- fast, convenient online submission

- thorough peer review by experienced researchers in your field

- rapid publication on acceptance

- support for research data, including large and complex data types

- gold Open Access which fosters wider collaboration and increased citations

- maximum visibility for your research: over $100 \mathrm{M}$ website views per year

At BMC, research is always in progress.

Learn more biomedcentral.com/submissions 